\title{
Disruptive natural selection by male reproductive potential prevents underexpression of the genes encoding proteins on the human Y chromosome as a self-domestication syndrome
}

\author{
Mikhail Ponomarenko \\ Systems Biology Department \\ Institute of Cytology and Genetics, ICG \\ SB RAS \\ Novosibirsk, Russia \\ pon@bionen.nsc.ru \\ Irina Chadaeva \\ Systems Biology Department \\ Institute of Cytology and Genetics, ICG \\ SB RAS \\ Novosibirsk, Russia \\ ichadaeva@bionet.nsc.ru
}

\author{
Dmitry Oshchepkov \\ Systems Biology Department \\ Institute of Cytology and Genetics, ICG \\ SB RAS \\ Novosibirsk, Russia \\ diman@bionet.nsc.ru \\ Dmitry Rasskazov \\ Systems Biology Department \\ Institute of Cytology and Genetics, ICG \\ SB RAS \\ Novosibirsk, Russia \\ rassk@bionen.nsc.ru
}

\author{
Alexander Osadchuk \\ Animal Genetics Department \\ Institute of Cytology and Genetics, ICG \\ SB RAS \\ Novosibirsk, Russia \\ osadchuk@bionet.nsc.ru \\ Ludmila Osadchuk \\ Animal Genetics Department \\ Institute of Cytology and Genetics, ICG \\ SB RAS \\ Novosibirsk, Russia \\ losadch@bionet.nsc.ru
}

\begin{abstract}
We performed an in silico genome-wide analysis of all SNPs located within $70 \mathrm{bp}$ proximal promoters in front of the all experimentally knowns starts of protein-coding transcripts from human $Y$ chromosome within the framework of the current release \#151 of the dbSNP database and GRCh38/hg38 assembly of the human reference genome, which are publicly available using the UCSC Genome Browser. As a result, we first found disruptive natural selection by male reproductive potential preventing underexpression of the $Y$ linked proteins under this study as if self-domestication would have happened during the human origing and evolution that could cause male fertility disorders as self-domestication syndrome.
\end{abstract}

Keywords - reproductive potential, human, Y chromosome, gene, promoter, TATA box, TATA-binding protein, singlenucleotide polymorphism, candidate SNP marker, verification

\section{Background}

In population ecobiology, the conception of reproductive potential define the most common vital indicator of a probability estimate to produce and regrow a healthy descendant until his/her reproductive maturity at the best conditions. This term associates lifespan and living conditions of an individual with one another, which correspond to his/her disease susceptibilities encoded by his/her genome and his/her both environment and lifestyle. Female reproductive potential seems to be most investigated in depth, width, and complexity now, whereas the male one has not subjected by equal amount of attention as yet. That is why, in this work we focused our attention on the only human $\mathrm{Y}$ chromosome as the male specific part of the human genome and, thus, predicted in silico candidate single-nucleotide polymorphism (SNP) markers of male reproductive potential within the framework of the current status of both reference human genome and variome.

\section{Materials and methods}

Using the current release \#151 of the dbSNP database [1] and GRCh38/hg38 assembly of the human reference genome [2] via publicly available Web-service UCSC Genome Browser [3] together with our earlier created Web-service
SNP TATA Comparator [4], we examined in silico all 1206 SNPs, none of which has been characterized so far regarding any link to human diseases (for brevity, hereinafter: unannotated SNPs) within $70 \mathrm{bp}$ proximal promoters of all 63 Y-linked genes.

\section{Results and Discussions}

As a result, we found 261 candidate SNP markers for male reproductive potential, which can statistically significantly change the binding affinity of TATA-binding protein (TBP) for these promoters. Among them, there are candidate SNP markers of spermatogenesis disorders (e.g., rs1402972626), pathoembryogenesis (e.g., rs28378830), adenovirus infection of spermatozoa leading to male infertility via spontaneous abortion (e.g., rs1317376848), azoospermia via testicular degeneration (e.g., rs1420856028), reduced sperm quality (e.g., rs1225019830), increased verbal IQ reducing male reproductive potential (e.g., rs1462000578), pediatric cancer (e.g., rs1483581212), as well as male anxiety damaging family relationships and mother's and children's health (e.g., rs187456378).

We first selectively verified in vitro both absolute and relative values of the analyzed TBP-promoter affinity using electrophoretic mobility shift assay (EMSA), where Pearson's simple linear correlation coefficients between our predictions and measurements were $r=0.84$ (significance $p<0.025$ ) and $r=0.98(p<0.025)$, respectively. Then, we found a twofold excess of the amount of candidate SNP markers increasing the TBP-promoter affinity relatively those for its decrease, whereas the genome-wide norm $[5,6]$ consists in four-fold excess of SNP-induced damages of TBP-promoter complexes in comparison with SNP-caused improvements of these complexes $[6,7]$ ( $p<0.05$ according to binomial distribution). This observed difference in the SNP occurrences in the entire genome as a whole and on chromosome $\mathrm{Y}$ in particular points to the natural selection against deficient male-specific expression of proteins that are encoded by this chromosome.

Simultaneously, the occurrences of candidate SNP markers, which can increase and decrease male reproductive potential, seem to be indistinguishable $(p<0.05)$ as if male self-domestication could have happened, with its experimentally known disruptive natural selection, according 
to Belyaev's observations made during foxes domestication [9].

Finally, because there is still not enough scientific evidence that this could have happened, we discuss the human diseases associated with candidate SNP markers of male reproductive potential in comparison with the known experimental data and natural observation on differences between wolfs and dogs [10], boars and pigs [11] as well as wild and domestic ducks [12], horses [13], mice [14]. Summing up, we found commonly accepted prototype traits of anthropogenic selection associated with animal domestication for the majority of candidate SNP markers of male reproductive potential except for the only candidate SNP markers for suicide (e.g., rs772325955) and verbal IQ (e.g., rs1393008234), which could be human-specific.

\section{Conclusions}

In general, our results obtained within the framework of this work indicate that a decrease in the reproductive potential in men can be a self-domestication syndrome as a manifestation of destructive natural selection against a deficiency of proteins encoded by the human Y chromosome.

\section{ACKNOWLEDGMENTS}

The study supervision were supported by project \#19-1500075 from the Russian Science Foundation (for MP and LO, $\mathrm{AO})$. The software development was financed by project \#0324-2019-0040-C01 from the Russian Government Budget (for DR). The data compilation was supported by project and \#18-34-00496 from Russian Foundation for Basic Research (for IC). The data analysis was supported by was supported by the Russian Federal Science \& Technology Program for the Development of Genetic Technologies (for DO). The in silico result interpretation was supported by by project \#0324-20190041-C01 from the Russian Government Budget (for AO).

\section{REFERENCES}

[1] S. Sherry, M. Ward., M. Kholodov, J. Baker, L. Phan, E. Smigielski, K. Sirotkin. "dbSNP: the NCBI database of genetic variation". Nucleic Acids Res. vol. 29, pp. 308-311, 2001.

[2] D. Zerbino, S. Wilder, N. Johnson, T. Juettemann, P. Flicek. "The Ensembl regulatory build”. Genome Biol. vol. 16: pp. 56. 2015.
[3] M. Haeussler, B. Raney, A. Hinrichs, H. Clawson, A. Zweig, D. Karolchik, J. Casper, M. Speir, D. Haussler, W. Kent. "Navigating protected genomics data with UCSC Genome Browser in a box". Bioinformatics. vol. 31. pp. 764-766. 2015.

[4] M. Ponomarenko, D. Rasskazov, O. Arkova, P. Ponomarenko, V. Suslov, L. Savinkova, N. Kolchanov. "How to use SNP TATA_Comparator to find a significant change in gene expression caused by the regulatory SNP of this gene's promoter via a change in affinity of the TATA-binding protein for this promoter". Biomed. Res. Int. vol. 2015. pp. 35983004625. 2015.

[5] J. Haldane. "The cost of natural selection". J Genet. vol. 55. pp. 511524. 1957.

[6] M. Kimura. "Evolutionary rate at the molecular level". Nature. vol. 217. pp. 624-626. 1968.

[7] M. Kasowski, F. Grubert, C. Heffelfinger, M. Hariharan, A. Asabere, S. Waszak, L. Habegger, J. Rozowsky, M. Shi, A. Urban, M. Hong, K. Karczewski, W. Huber, S. Weissman, M. Gerstein, J. Korbel, M. Snyder M. 2010. "Variation in transcription factor binding among humans". Science. vol. 328. pp.: 32-235. 2010

[8] 1000 Genomes Project Consortium, G. Abecasis, A. Auton, L. Brooks, M. DePristo, R. Durbin, R. Handsaker, H. Kang, G. Marth, G. McVean. "An integrated map of genetic variation from 1.092 human genomes". Nature. vol. 491. pp. 56-65. 2012.

[9] D. Belyaev. "The Wilhelmine E. Key 1978 invitational lecture. Destabilizing selection as a factor in domestication". J Hered. vol. 70. pp. 301-308. 1979.

[10] C. Theofanopoulou, S. Gastaldon, T. O'Rourke, B. Samuels, P. Martins, F. Delogu, S. Alamri, C. Boeckx. "Self-domestication in Homo sapiens: insights from comparative genomics". PLoS One. vol. 12. pp. e0185306. 2017.

[11] F. Almeida, M. Leal, L. Franca. "Testis morphometry, duration of spermatogenesis, and spermatogenic efficiency in the wild boar (Sus scrofa scrofa). Biol Reprod. vol. 75. pp. 792-799. 2006.

[12] A. Charuta, H. Markowska-Pliszka, B. Bartyzel, J.Wysocki. Size of heart of the domestic Pekin duck (Anas platyrhynchosf. domestica) and wild duck (Anas platyrhynchos, Linnaeus, 1758). Acta Sci Pol Med Veterinaria. vol. 4. pp. 11-19. 2005.

[13] P. Librado, C. Gamba, C. Gaunitz, C. Der Sarkissian, M. Pruvos, A Albrechtsen, A. Fages, N. Khan, M. Schubert, V. Jagannathan, A. Serres-Armero, L. Kuderna, I. Povolotskaya, A. Seguin-Orlando, S. Lepetz, M. Neuditschko, C. Theves, S. Alquraishi, A. Alfarhan, K. AlRasheid, S. Rieder, Z. Samashev, H. Francfort, N. Benecke, M. Hofreiter, A. Ludwig, C. Keyser, T. Marques-Bonet, B. Ludes, E. Crubezy, T. Leeb, E. Willerslev, L. Orlando. "Ancient genomic changes associated with domestication of the horse". Science. vol. 356. pp. 442-445. 2017.

[14] D. Blanchard, G. Griebel, R. Blanchard. "Mouse defensive behaviors: pharmacological and behavioral assays for anxiety and panic". Neurosci Biobehav Rev. vol. 25. pp. 205-218. 2001. 